\title{
Rehabilitation after total hip arthroplasty: Is there an evidence?
}

\author{
Aprato $A^{*}$, Baroni $C$ and Massè $C$ \\ Department of Orthopaedics, Traumatology and Rehabilitation, University of Torino, Torino, Italy
}

Osteoarthritis is the most common joint disorder worldwide and a leading cause of pain and disability prevalent among middle-aged and elderly adults, impacting many health outcomes [1-2].

Total hip arthroplasty (THA) is the gold standard treatment of end-stage arthritis and the number of person who undergo THA has increased significantly over the last few years with the aging of the population.

The success of THA is due to its predictable pain relief, functional recovery and improvement in quality of life facilitating the patient's return to activities of daily living (ADLs) and even to labor activities [3].

Following surgery, physiotherapist led exercise-based rehabilitation is often prescribed [4,5] to optimize functional recovery. Several protocols have been proposed and used in clinical practice; all protocols include hip-joint mobilization with low-resistance weights to strengthen the muscles, gait and strength training. A good level of evidence supports such prescriptions: physiotherapy programs showed better results when compared to no or minimal intervention following THA. Several studies showed how patients who received THA without a rehabilitation program will develop functional limitations [6,7]. A recent systematic review concluded that early multidisciplinary rehabilitation improved outcomes at the level both of activity and participation [8].

Furthermore, Chou [9] showed how early rehabilitation can significantly reduce the incidence of prosthetic infection, luxation and thromboembolic disease total rehabilitation, medical expense and number of outpatient visits.

However, the overall effectiveness of physiotherapy remains uncertain [4]: it is unclear if clinic-based one-to-one or group-based programs are superior over monitored or unmonitored home-based programs for the recovery of pain, range of motion and quality of life after THA. Furthermore, there is no consensus about how soon rehabilitation should start after surgery and what benefits it might bring to the patients. In everyday practice is common to find differences in exercise type, specific equipment, duration of each session and interval between sessions [10].

There is no strong evidence that suggests how to decide when rehabilitation intervention is appropriate and whether different rehabilitation intervention and different timing may impact on patient's outcome outcome of THA. Moreover, the optimal intensity, frequency and effects of rehabilitation and associated social costs are unknown: randomized trials are difficult to undertake and no evidence-based guidelines are available worldwide.

Although there is still no routine rehabilitation protocol worldwide admitted after THA, it is clear that physiotherapy is mandatory for all patient that have a modified hip anatomy prior to surgery, as in trauma patient (acetabular or femoral fractures), hip dysplasia or secondary osteoarthritis (slipped capital femoral epiphysis etc). Those patients have muscular and soft tissue disorders and proprioceptive deficit that may cause relevant stiffness, deformity, loss of movement and walking difficulty. Therefore, in these cases, a proper pre and post-op rehabilitation protocol is needed to restore the normal function. Many authors suggest that preoperative loss of weight, muscle strengthening and a post-op early mobilization with active exercises to increase hip range of motion such as adductor stretching, may play a key role in the rehabilitation process but again the recommendation are not supported by strong evidence.

In conclusion, in literature the evidence is poor about rehabilitation in patients who had undergone THA and there is a huge need of high quality studies on this topic.

\section{References}

1. Sharma L, Kapoor D, Issa S (2006) Epidemiology of osteoarthritis: An update. Curr Opin Rheumatol 18: 147-156. [Crossref]

2. Alviar MJ, Olver J, Brand C, Hale T, Khan F (2011) Do patient-reported outcome measures used in assessing outcomes in rehabilitation after hip and knee arthroplasty capture issues relevant to patients? Results of a systematic review and ICF linking process. J Rehabil Med 43: 374-381. [Crossref]

3. Freburger JK (2000) An analysis of the relationship between the utilization of physical therapy services and outcomes of care for patients after total hip arthroplasty. Phys Ther 80: 448-458. [Crossref]

4. Coulter CL, Scarvell JM, Neeman TM, Smith PN (2013) Physiotherapist-directed rehabilitation exercises in the outpatient or home setting improve strength, gait speed and cadence after elective total hip replacement: A systematic review. J Physiother 59: 219-226. [Crossref]

5. Brotzman SB, Manske RC (2011) Clinical Orthopaedic Rehabilitation: An EvidenceBased Approach. 3rd. Philadelphia, Pa, USA: Elsevier Mosby.

6. Trudelle-Jackson E, Smith SS (2004) Effects of a late-phase exercise program after total hip arthroplasty: A randomized controlled trial. Arch Phys Med Rehabil 85: 10561062. [Crossref]

7. Okoro T, Lemmey AB, Maddison P, Andrew JG (2012) An appraisal of rehabilitation regimes used for improving functional outcome after total hip replacement surgery. Sports Med Arthrosc Rehabil Ther Technol 4: 5. [Crossref]

8. Khan F, Ng L, Gonzalez S, Hale T, Turner-Stokes L (2008) Multidisciplinary rehabilitation programmes following joint replacement at the hip and knee in chronic arthropathy. Cochrane Database Syst Rev 2: CD004957. [Crossref]

${ }^{*}$ Correspondence to: Alessandro Aprato, Department of Orthopaedics, Traumatology and Rehabilitation, University of Torino, Torino, Italy, E-mail: ale_aprato@hotmail.com

Received: August 18, 2018; Accepted: August 29, 2018; Published: August 31, 2018 
Aprato A (2018) Rehabilitation after total hip arthroplasty: Is there an evidence?

9. Chiung-Jui Su D, Yuan K, Weng S, Hong R, Wu M, et al. (2015) Can Early Rehabilitation after Total Hip Arthroplasty Reduce Its Major Complications and Medical Expenses? Report from a Nationally Representative Cohort. Biomed Res Int 2015: 641958.
10. Di Monaco M, Vallero F, Tappero R, Cavanna A (2009) Rehabilitation after total hip arthroplasty: A systematic review of controlled trials on physical exercise programs. Eur J Phys Rehabil Med 45: 303-317. [Crossref]

Copyright: (C2018 Aprato A. This is an open-access article distributed under the terms of the Creative Commons Attribution License, which permits unrestricted use, distribution, and reproduction in any medium, provided the original author and source are credited. 